\title{
Atmospheric water droplets can catalyse atom pair break-up via surface-induced resonance repulsion
}

Mathias Boström, Clas Persson, Drew F. Parsons, Simen A. Ellingsen and Bo Sernelius

\section{Linköping University Post Print}

\section{Tweet}

N.B.: When citing this work, cite the original article.

Original Publication:

Mathias Boström, Clas Persson, Drew F. Parsons, Simen A. Ellingsen and Bo Sernelius, Atmospheric water droplets can catalyse atom pair break-up via surface-induced resonance repulsion, 2013, Europhysics letters, (101), 4. http://dx.doi.org/10.1209/0295-5075/101/43002

Copyright: EDP Sciences

http://publications.edpsciences.org/

Postprint available at: Linköping University Electronic Press http://urn.kb.se/resolve?urn=urn:nbn:se:liu:diva-90764 


\title{
Atmospheric water droplets can catalyse atom pair break-up via surface-induced resonance repulsion
}

\author{
Mathias Boström ${ }^{1,2}$, Clas Persson ${ }^{1,3}$, Drew F. Parsons ${ }^{2}$, Simen Å. Ellingsen ${ }^{4}$ and Bo E. Sernelius $^{5}$ \\ ${ }^{1}$ Department of Materials Science and Engineering, Royal Institute of Technology - SE-100 44 Stockholm, \\ Sweden, EU \\ 2 Department of Applied Mathematics, Australian National University - Canberra, Australia \\ ${ }^{3}$ Department of Physics, University of Oslo - P. O. Box 1048 Blindern, NO-0316 Oslo, Norway \\ ${ }^{4}$ Department of Energy and Process Engineering, Norwegian University of Science and Technology, \\ NO-7491 Trondheim, Norway \\ ${ }^{5}$ Division of Theory and Modeling, Department of Physics, Chemistry and Biology, Linköping University, \\ SE-581 83 Linköping, Sweden, EU
}

received 7 November 2012; accepted in final form 30 January 2013

published online 28 February 2013

PACS 34.20.Cf - Interatomic potentials and forces

PACS 42.50.Lc - Quantum fluctuations, quantum noise, and quantum jumps

PACS 92.60. Jq - Water in the atmosphere

\begin{abstract}
We present the theory for a retarded resonance interaction between two identical atoms near a dielectric surface. In free space the resonance interaction between isotropically excited atom pairs is attractive at all atom-atom separations. We illustrate numerically how this interaction between oxygen, sulphur, hydrogen, or nitrogen atom pairs may turn repulsive near water droplets. The results provide evidence of a mechanism causing excited state atom pair breakage to occur in the atmosphere near water droplets.

Copyright (c) EPLA, 2013
\end{abstract}

Two identical atoms, one isotropically excited and the other in its ground state, can be bound together in free space due to an attractive retarded resonance interaction [1-4]. Interestingly, the same retarded resonance interaction can influence the binding energy of atom pairs $[5,6]$. There have been both experimental [7] and theoretical [8] evidence for the enhancement of dipoledipole interactions in microcavities. We have recently predicted a way by which very large molecules may form by resonance interaction between two identical atoms in a narrow cavity [9]. A problem of significance to catalysis is how breakage and formation of molecules may occur near surfaces $[10,11]$. We propose a way that excited state atom pairs in the atmosphere may be split near water drops. It derives from the retarded resonance interaction between a pair of identical atoms, one being isotropically excited and the other in its ground state. In free space this interaction is attractive but near a dielectric surface it may under specific circumstances provide enough repulsive energy to split atom pairs.

The aim of this letter is to present the theory for retarded resonance interaction between a pair of identical atoms in a collective excited state near a dielectric surface. As an illustrative example we consider identicalatom pairs (oxygen, sulphur, nitrogen and hydrogen) near an air-water interface. This may be a model for interactions between atoms photodissociated from gas molecules present in the atmosphere $[12,13]$, when they come near surfaces of water droplets. For this reason we choose water to be our surface of choice for numerical examples, although our results are changed only quantitatively by insertion of other surfaces relevant in different applications, such as bodies of metal or silica frequently used in experiments and nanomechanical settings, into the same governing equations. The mechanism is also relevant to the study of helium dimers. Helium has a particularly long-lived excited state with a lifetime of several hours [14] which is observed experimentally to form giant, weakly bound dimers [15].

When the two atoms are far from the surface there is an attractive resonance interaction which vanishes when retardation is neglected. A schematic illustration of the system is shown in fig. 1. The resonance interaction between atom pairs in the atmosphere, attractive in free 


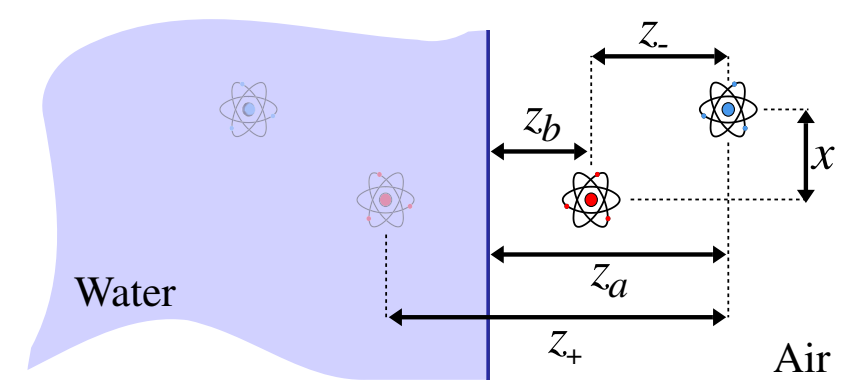

Fig. 1: (Colour on-line) Two atoms in vacuum (air) near a water surface. Also shown are their mirror images.

space, will turn repulsive sufficiently close to a water droplet, depending on the separation and alignment of the atoms relative to the surface.

The diagonal matrix elements of the susceptibility needed to calculate the retarded resonance interaction is obtained from the Green's function matrix given in the literature $[16,17]$. A contribution to bond splitting between atom pairs is predicted to occur due to excitation-induced resonance interaction between atoms which becomes repulsive sufficiently near the surface of a water droplet. Once the atom pairs are broken the radicals are free to interact and form new constellations with other atoms or molcules.

We first recall the standard argument [1,3,4]: Consider two identical atoms where one initially is in its ground state and the other is in an excited state. This state can also be represented by a superposition of states: one symmetric and one antisymmetric with respect to interchange of the atoms. While the symmetric state will quickly decay into two ground-state atoms, the antisymmetric state can be quite long-lived. The system can thus be trapped in the antisymmetric state $[1,3]$. The energy migrates back and forth between the two atoms until either the two atoms move apart or a photon is emitted away from the system. First-order dispersion interactions are caused by this coupling of the system, i.e., the energy difference between the two states is separation $(d)$ dependent. After writing down the equations of motion for the excited system it is straightforward to derive the zero-temperature Green's function for two identical and isotropic atoms $[1,4]$. We use CGS units in all equations. The resonance frequencies of the system are given by the following equation [1]:

$$
1-\alpha(1 \mid \omega) \alpha(2 \mid \omega) \mathbf{T}(d \mid \omega)^{2}=0,
$$

where $\alpha$ is the atomic polarizability and $\mathbf{T}$ the susceptibility tensor. Atomic polarizabilities were calculated over imaginary frequencies by TURBOMOLE [18] using DFT quantum chemical methods with the PBE0 functional [19]. The augmented correlation consistent basis set aug-ccpV5Z [20-22] was employed. Polarizabilities were calculated for the ground state with highest spin, that is the doublet state for $\mathrm{H}$, triplet state for $\mathrm{O}$ and $\mathrm{S}$, and

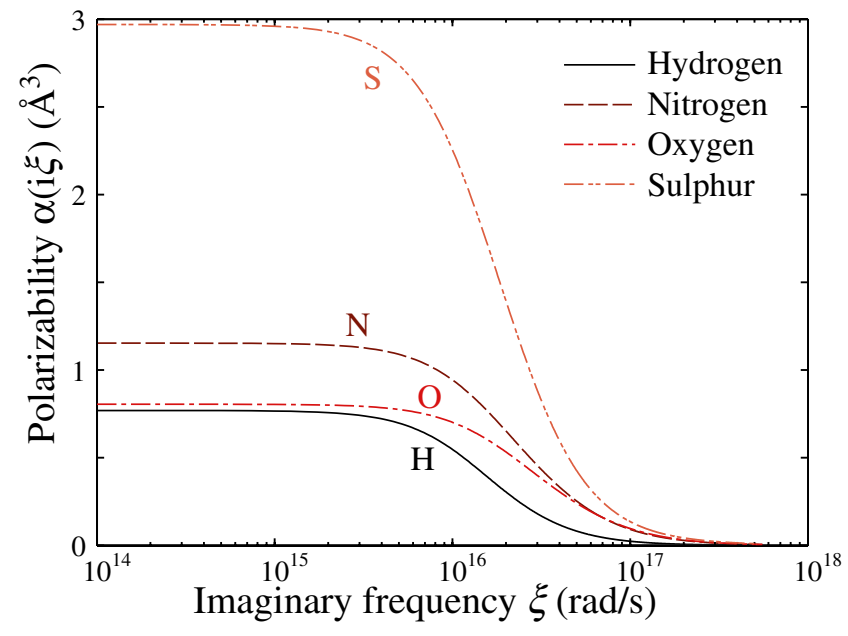

Fig. 2: (Colour on-line) The polarizabilities (for imaginary frequencies) of sulfur, oxygen, hydrogen and nitrogen atoms.

quadruplet state for N. Figure 2 shows the numerically evaluated polarizabilities of oxygen, sulphur, nitrogen and hydrogen atoms used in this study.

When the atoms are identical the resonance condition, eq. (1), can be separated into an antisymmetric and a symmetric part. The symmetric state decays quickly to two ground-state atoms, but the system can be trapped in an antisymmetric state from which no dipole transition to the ground state exists [1].

The resonance interaction energy of such a system is

$$
U(d)=\hbar\left[\omega_{r}(d)-\omega_{r}(\infty)\right] .
$$

Note that spatially oscillating potentials, which have been subject to much debate [1], are not relevant in the long time limit [23]. The relevant solution of eq. (1) is to be understood as a pole of the antisymmetric part of the underlying Green's function. With standard use of the Cauchy integral theorem [24] we can express it as a contour integral encircling the pole in question to obtain a simple expression for the resonance interaction energy,

$$
U(d) \simeq(-\hbar / \pi) \int_{0}^{\infty} \mathrm{d} \xi \alpha(1 \mid i \xi) \operatorname{Tr} \mathbf{T}(d \mid i \xi) .
$$

$\operatorname{Tr} \mathbf{T}$ is the trace of the susceptibility tensor.

To account for the temperature $(T)$ dependence we simply replace the integration over imaginary frequencies by a summation over discrete Matsubara frequencies [24],

$$
\frac{\hbar}{2 \pi} \int_{0}^{\infty} \mathrm{d} \xi \rightarrow k_{\mathrm{B}} T \sum_{n=0}^{\infty \prime}, \quad \xi_{n}=2 \pi n k_{\mathrm{B}} T / \hbar,
$$

where $k_{\mathrm{B}}$ is the Boltzmann constant and the prime indicates that the $n=0$ term should be divided by 2 .

We consider the case when $x$ is the distance between the two atoms parallel to the surface and $z_{a}$ and $z_{b}$ are the distances from atom $a$ and $b$ to the surface, as shown 


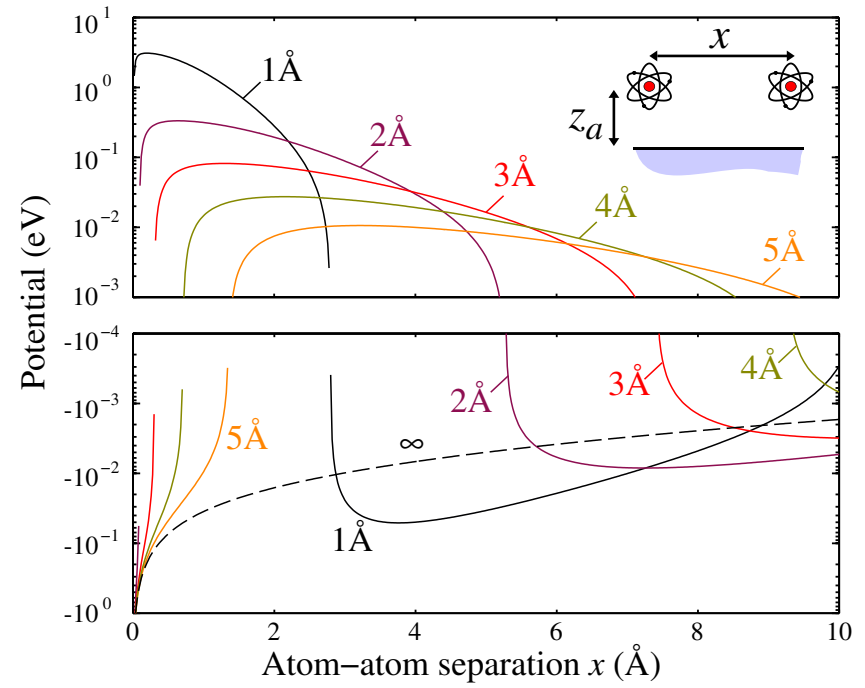

Fig. 3: (Colour on-line) The retarded resonance interaction between two sulfur atoms in an isotropically excited state $\left(z_{a}=z_{b}\right.$ ranging from $1 \AA$ to $5 \AA$ from top to bottom in steps of $1 \AA$ ) as a function of distance $(x)$ between the atoms parallel to the water surface. The potential between two atoms in free space is shown for comparison (dashed line).

in fig. 1. The susceptibility tensor is a sum of terms from free space susceptibility $\left(T_{i i}^{0}\right)$, plus terms from $p\left(T_{i i}^{1 p}\right)$ and $s\left(T_{i i}^{1 s}\right)$ surface corrections due to the presence of a surface. The $s$ - and $p$-terms are the results from coupling between $s$ - and $p$-polarized waves, respectively. In free space the susceptibility matrix, $\mathbf{T}\left(\rho \mid i \xi_{n}\right)$, has the following: diagonal matrix elements:

$$
\begin{aligned}
& T_{x x}^{0}=\left(A-B x^{2} / d^{2}\right) e^{-\xi_{n} d / c} / d^{3}, \\
& T_{y y}^{0}=A e^{-\xi_{n} d / c} / d^{3}, \\
& T_{z z}^{0}=\left[A-B z_{m}^{2} /\left(d^{2}\right)\right] e^{-\xi_{n} d / c} / d^{3},
\end{aligned}
$$

where

$$
\begin{aligned}
& A=1+\left(d \xi_{n} / c\right)+\left(d \xi_{n} / c\right)^{2}, \\
& B=3+\left(3 d \xi_{n} / c\right)+\left(d \xi_{n} / c\right)^{2}
\end{aligned}
$$

and $d=\sqrt{x^{2}+z_{-}^{2}}$. We have defined $z_{+}=z_{a}+z_{b}$ and $z_{-}=$ $z_{a}-z_{b}$ with the first being the distance between atom $a$ and the image of atom $b$ inside the surface and the second being distance between the two atoms.

The corresponding surface-induced corrections to the susceptibility matrix $[16,17]$ have contributions from $p$ and $s$ polarizations,

$$
\begin{aligned}
T_{x x}^{1 s} & =\frac{\xi_{n}^{2}}{2 c^{2}} \int_{0}^{\infty} \frac{q \mathrm{~d} q}{\gamma_{0}} e^{-\gamma_{0} z_{+}} r_{s}\left[J_{0}(q x)+J_{2}(q x)\right] \\
T_{y y}^{1 s} & =\frac{\xi_{n}^{2}}{2 c^{2}} \int_{0}^{\infty} \frac{q \mathrm{~d} q}{\gamma_{0}} e^{-\gamma_{0} z_{+}} r_{s}\left[J_{0}(q x)-J_{2}(q x)\right] \\
T_{z z}^{1 s} & =0
\end{aligned}
$$

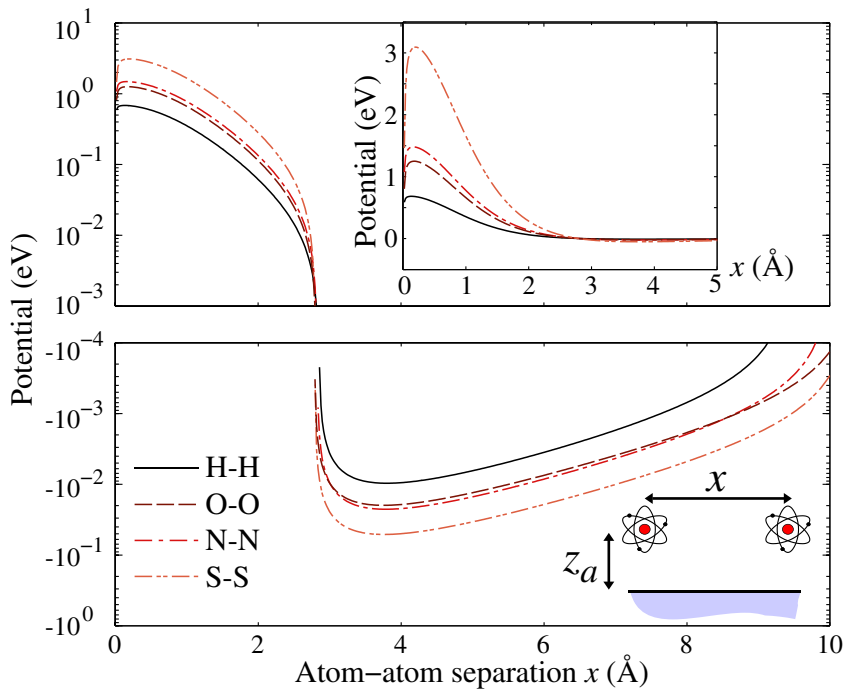

Fig. 4: (Colour on-line) The retarded resonance interaction between excited pairs of different atom species (S-S, N-N, H-H, and $\mathrm{O}-\mathrm{O})$ as a function of distance between the atoms parallel to the water surface, $z_{a}=z_{b}=1 \AA$. Inset: the same in linear scale.

and

$$
\begin{aligned}
& T_{x x}^{1 p}=-\frac{1}{2} \int_{0}^{\infty} q \mathrm{~d} q \gamma_{0} e^{-\gamma_{0} z_{+}} r_{p}\left[J_{0}(q x)-J_{2}(q x)\right], \\
& T_{y y}^{1 p}=-\frac{1}{2} \int_{0}^{\infty} q \mathrm{~d} q \gamma_{0} e^{-\gamma_{0} z_{+}} r_{p}\left[J_{0}(q x)+J_{2}(q x)\right], \\
& T_{z z}^{1 p}=-\int_{0}^{\infty} \mathrm{d} q \frac{q^{3}}{\gamma_{0}} e^{-\gamma_{0} z_{+}} r_{p} J_{0}(q x),
\end{aligned}
$$

respectively.

Surface reflection coefficients are

$$
r_{s}=\frac{\gamma_{0}-\gamma_{1}}{\gamma_{0}+\gamma_{1}}, \quad r_{p}=\frac{\epsilon \gamma_{0}-\gamma_{1}}{\epsilon \gamma_{0}+\gamma_{1}}
$$

where $\gamma_{0}=\sqrt{q^{2}+\xi_{n}^{2} / c^{2}}$ and $\gamma_{1}=\sqrt{q^{2}+\varepsilon\left(i \xi_{n}\right) \xi_{n}^{2} / c^{2}}$. In the non-retarded limit the resonance interaction between a pair of identical atoms vanishes in free space. The corresponding retarded result in free space is [1] $U(d)=$ $-2 \hbar c \alpha(0) /\left(\pi d^{4}\right)$. This result is obtained when using the so called London approximation for the atomic polarizability [25]. It is interesting to note that this asymptote has the same power-law as the Casimir-Polder energy between a ground-state atom and a perfectly conducting surface [26], $U^{\mathrm{CP}}(z)=-3 \hbar c \alpha(0) /\left(8 \pi z^{4}\right)$.

We will now proceed to a numerical calculation of the retarded resonance interaction at $300 \mathrm{~K}$ between pairs of sulphur, oxygen, hydrogen and nitrogen atoms near the surface of a water droplet. All distances being small compared to the size of the water droplet, we can treat the water droplet as a planar air-water interface. Figure 3 shows the retarded resonance interaction between two sulphur atoms situated at the same distance from an air-water interface. We observe that as $z_{a}$ decreases the interaction turns more and more repulsive 

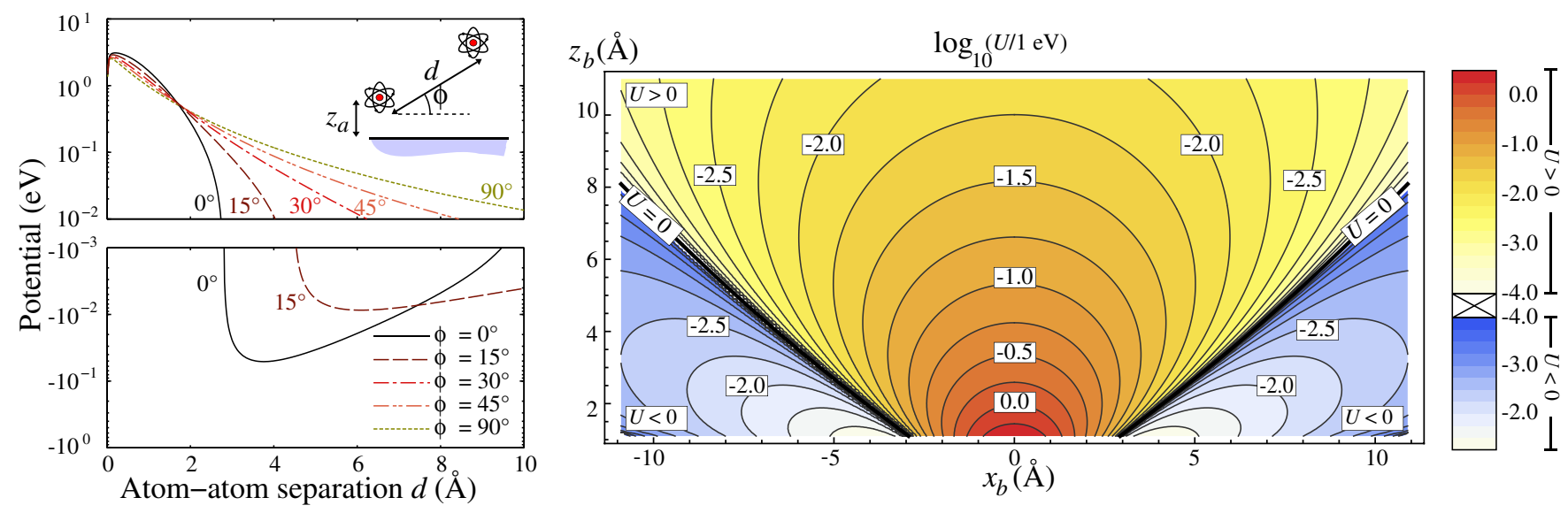

Fig. 5: (Colour on-line) The retarded resonance interaction between an excited pair of sulfur atoms having different orientations relative to the surface. Atom $a$ is in a fixed position at $x_{a}=0, z_{a}=1 \AA$. Left: potential for different angles of approach. Right: logarithmic contour plot of the potential landscape "seen" by atom $b$. The thick lines show $U=0$ with $U>0$ above and $U<0$ below it, respectively.

due to the influence of $p$-polarized surface corrections. The corresponding results for different atom pairs at a fixed distance to the water droplet are shown in fig. 4 . These two figures are particularly illustrative since the interaction between the atoms and the surface are the same leaving a cetera paribus view of the surface-induced effect on the atom-atom resonance interaction.

Consider finally the retarded resonance interaction between two sulphur atoms at different orientations relative to the surface. Now the influence on the two atoms by the surface is different. The result is shown in fig. 5, where we fix atom $a$ close to the wall and vary the position of atom $b$. We observe that the repulsive force (potential gradient) between the atoms is strongest when the atoms are aligned parallel to the surface, and weakest when alignment is orthogonal to it. Only when the angle of alignment with respect to the surface is small are bound states still found to be possible, whereas in the orthogonal direction the interaction is repulsive for all important separations. Furthermore it is evident that unlike the ground-state Casimir-Polder interaction considered in refs. $[16,17]$, the attraction between two atoms near a surface is not increased for any orientation when the atoms are very close.

We have discussed how the presence of a surface influences the retarded resonance interaction between two identical atoms when one is in an isotropically excited state and the other in its ground state. As an example we chose the surface of a water droplet and atoms which are prevalent in the atmosphere. The choice of surface material is not essential to the results, and one might have chosen a metallic surface (for contact with many laboratory setups) or a material used in nano- and micro-scale engineering, such as silica, without any qualitative change to the results we obtain. The interaction between a pair of collectively and isotropically excited atoms near a water droplet can produce a repulsive energy between the two of the same order of magnitude as the bonding energy of diatomic molecules. This would affect the rate of dissociation of molecules near surfaces [11] to some extent, since decay via this channel is rendered less probable due to the additional energy required. But on the other hand, once a molecule is dissociated, the resonance repulsion provides a barrier which will reduce the rate of recombination. We may thereby envisage that where photodissociation might take place at some small distance from a water surface, the dissociated radicals can then be stabilized (i.e., recombination impeded) by moving towards the surface.

We investigated the directional dependence of the interaction between such a pair of collectively and isotropically excited atoms and found that the greatest repulsive force is found when the atoms are aligned parallel with the surface. However, only in close to parallel alignments are weakly bound states still possible, of the kind that were predicted by Stephen many years ago [3] for atoms in free space. When such a weakly bound pair gets close to a surface, i.e., that of a water droplet in the atmosphere, the repulsion demonstrated in this letter will more than likely cause the pair to break up. We predict that the concentration ratios $[\mathrm{M}] /[\mathrm{M} 2]$ for $\mathrm{M}=\mathrm{H}, \mathrm{N}, \mathrm{O}$, or $\mathrm{S}$ depend in part through the resonance repulsion on the presence of water droplets. This should be possible to detect experimentally. Atomic finite-size effects should come into play for very small atom-atom separations. The correction would to lowest order show up in a quadrupole term in the atomfield interaction, where the standard theory truncates at the dipole term. For a Rydberg atom above a plane metal surface quadrupole corrections were surprisingly small $(1 \%$ level) even when the atom-wall separation was of the same order as the radius of the Rydberg atom $(\sim 1 \mu \mathrm{m})$ [27]. That only gives an indication, though, not proof, that finite-size corrections are small. We will in the near future 
exploit the powerful formalism developed by Mahanty and Ninham [28] to consider finite-size effects on the resonance interaction.

$$
* * *
$$

CP and MB acknowledge support from VR (Contract No. C0485101) and STEM (Contract No. 34138-1). BES acknowledges financial support from VR (Contract No. 70529001). DFP acknowledges the support from the Australian Research Council. We thank BARRY W. NINHAM for stimulating discussions and comments. This work was supported by the NCI National Facility at the Australian National University.

\section{REFERENCES}

[1] Boström M., Longdell J. J., Mitchell D. J. and Ninham B. W., Eur. Phys. J. D, 22 (2003) 47.

[2] Sherkunov Y., Phys. Rev. A., 75 (2007) 012705.

[3] Stephen M. J., J. Chem. Phys., 40 (1964) 669.

[4] Mclachlan A. D., Mol. Phys., 8 (1964) 409.

[5] Jones K. M., Julienne P. S., Lett P. D., Phillips W. D., Tiesinga E. and Williams C. J., Europhys. Lett., 35 (1996) 85.

[6] Mcalexander W. I., Abraham E. R. I. and Hulet R. G., Phys. Rev. A, 54 (1996) 54.

[7] Hopmeier M., Guss W., Deussen M., Göbel E. O. and Mahrt R. F., Phys. Rev. Lett., 82 (1999) 4118.

[8] Agarwal G. S. and Dutta Gupta S., Phys. Rev. A., 57 (1998) 667.

[9] Boström M., Brevik I., Sernelius Bo E., Dou M., Persson C. and Ninham B. W., Phys. Rev. A., 86 (2012) 014701.

[10] Blum Z., Hyde S. T. and Ninham B. W., J. Phys. Chem., 97 (1993) 661.
[11] Darling G. R. and Holloway S., Rep. Prog. Phys., 58 (1995) 1595.

[12] Turco R. P., Geophy. Surv., 2 (1975) 153.

[13] Crutzen P., Can. J. Chem., 52 (1974) 1569.

[14] Baldwin K. G. H., Contemp. Phys., 46 (2005) 105.

[15] Léonard J., Walhout M., Mosk A. P., Müller T., Leduc M. and Cohen-Tannoudji C., Phys. Rev. Lett., 91 (2003) 073203.

[16] Safari H., Buhmann S. Y., Welsch D. G. and Dung H. T., Phys. Rev. A., 74 (2006) 042101.

[17] Buhmann S. Y., Safari H., Dung H. T. and Welsch D. G., Opt. Spectrosc., 103 (2007) 374.

[18] TURBOMOLE V6.4 2012, a development of University of Karlsruhe and Forschungszentrum Karlsruhe GmbH, 1989-2007, Turbomole GmbH, since 2007; available from http://www . turbomole.com.

[19] Adamo C., Cossi M., Scalmani G. and Barone V., Chem. Phys. Lett., 307 (1999) 265.

[20] Dunning T. H., J. Chem. Phys., 90 (1989) 1007.

[21] Woon D. E. and Dunning T. H., J. Chem. Phys., 98 (1993) 1358.

[22] Peterson K. A. and Dunning T. H., J. Chem. Phys., 117 (2002) 10548.

[23] Haakh H., Schiefele J. and Henkel C., Int. J. Mod. Phys.: Conf. Ser., 14 (2012) 347.

[24] Sernelius Bo E., Surface Modes in Physics (Wiley-VCH, Berlin) 2001.

[25] London F., Z. Phys. Chem B,, 11 (1930) 222.

[26] Casimir H. B. G. and Polder D., Phys. Rev., 73 (1948) 360.

[27] Crosse J. A., Ellingsen S. A., Clements K., Buhmann S. Y. and Scheel S., Phys. Rev. A., 82 (2010) 010901.

[28] Mahanty J. and Ninham B. W., Dispersion Forces (Academic Press, London) 1976. 